\title{
Relativity principle in coordinate free presentation
}

\section{Rylov Y.A.}

Institute for Problems in Mechanics, Russian Academy of Sciences;

E-mail: Rylov<rylov@ipmnet.ru>;

It is shown, that the relativity principle can be presented without a reference to coordinate transformation, if the spacetime geometry is described in terms of the world function.

Keywords: relativity principle; metric approach; multivariant geometry.

DOI: $10.18698 / 2309-7604-2015-1-447-453$

The relativity theory has arisen in the beginning of the twentieth century as a result of negative result of measurement of the Earth velocity with respect to the ether $[1,2]$. This experiment generates a transition from the nonrelativistic physics to the relativistic one. After numerous discussions the scientific community accept the relativity principle. The principle of relativity means the requirement that the equations describing the laws of physics have the same form in all admissible frames of reference. It looks as a general physical principle, which concerns the event space arrangement and dynamics of physical bodies. Besides, formulation of the relativity principle contains a reference to coordinate systems and to the laws of their transformation. The reference to the way of description (frames of reference) looks rather strange in the formulation of a physical principle. Any physical principle is to admit a formulation, which does not contain a reference to the means of description. The fact, that such an important physical principle is not formulated in the coordinate free form, is a defect of our understanding of the relativity nature. We try to find a coordinate free formulation of the relativity principle.

Note, that in the first formulation of Einstein the relativity principle concerns only dynamics and dynamical equations. Minkowski showed that the principle of relativity concerns also the event space (space-time). Now the principle of relativity is considered as conditioned by the space-time properties. Unfortunately, conventional description of the contemporary space-time geometry begins from introduction of a coordinate system. Coordinate free description of the space-time geometry is absent in the axiomatic conception, which is used practically in all papers. Coordinate free description of geometry exists only in the framework of the metric conception of geometry [3].

The relativity principle means essentially that space and time have equal rights. Space may transform to time and vice versa. Space and time were different entities primordially. The relativity principle declares their connection. But the difference between the space and the time was primary, 
whereas their connection was secondary. It is possible another approach, when connection between the space and time is primary, whereas their difference is secondary. In this case the space-time geometry is a monistic conception, described by a unique structure: the space-time interval $\rho(P$, $Q$ ) between any two events (points) $P$ and $Q$ of the event space. To divide space and time one may introduce a second space-time structure $T(P, Q)$, describing temporal interval between any two events $P$ and $Q$ of the event space. In this case an absolute simultaneity appears. In this case we have the fortified geometry, i.e. a space-time geometry with two space-time structures. Having the two structures $\rho$ and $T$, one can introduce absolute spatial distance $S(P, Q)$ between any two events (points) $P$ and $Q$.

The relativity principle means, that there is only one space-time structure $\rho$ in the real event space. It is the coordinate free formulation of the relativity principle. Such a formulation is possible, if one uses the metric conception of the space-time geometry. Only in this case one can speak about one structure of the space-time geometry. In the conventional presentation of the space-time geometry there are several independent fundamental quantities, describing the spacetime geometry: (1) the metric dimension $D$, (2) linear vector space $L$ and coordinate system $K$, (3) space-time interval $\rho$ or the world function $\sigma=\frac{1}{2} \rho^{2}$.

In this case it is impossible to calculate the number of structures, which are used for description of the space-time geometry, because the enumerated quantities are not independent in reality. It is impossible to determine the real number of fundamental quantities. There is such a representation of the space-time geometry, where all geometric quantities and geometrical objects can be described in terms of one quantity: space-time metric $\rho$. This representation is realized at the metric approach to geometry.

Now the most general space-time geometry is the (pseudo) Riemannian geometry $G_{R}$. It is constructed as a deformation of the proper Euclidean geometry $G_{E}$. On a $D$-dimensional manifold there is the proper Euclidean geometry $G_{E}$, which is given by its world function $\sigma_{E}(P, Q)$ between two infinitesimally close points $P$ and $Q$

$$
\sigma_{E}(P, Q)=\sigma_{E}(x+d x, x)=g_{E i k} d x^{i} d x^{k}, \quad g_{E i k}=\operatorname{sgn}(1,1, \ldots 1)
$$

The $D$-dimensional Riemannian geometry arises after replacement Euclidean metric tensor $g_{E i k}$ by the Riemannian metric tensor $g_{i k}$. Such a replacement means a deformation of Euclidean 
geometry $G_{E}$. In other words, the Euclidean world function $\sigma_{E}$ is replaced by the Riemannian world function $\sigma_{R}$. Such a replacement is made on the same manifold, in the same coordinate system for world function of infinitesimally close points.

Unfortunately, infinitesimal world function (i.e. world function of infinitesimally close points) does not determine the geometry completely. The topology is important for definition of the geometry. For instance, geometry is different for two-dimensional Euclidean plane $P_{E 2}$ and for cylinder $C_{E 2}$, which is obtained from $P_{E 2}$ as a result of identification of points with coordinates $(x-L, y)$ and points with coordinates $(x+L, y)$. Metric tensors and are the same in $P_{E 2}$ and in $C_{E 2}$, but the world functions are different

$$
\begin{gathered}
P_{E 2}: 2 \sigma_{P 2}\left(x, y ; x^{\prime}, y^{\prime}\right)=\left(x-x^{\prime}\right)^{2}+\left(y-y^{\prime}\right)^{2} \\
E 2: 2 \sigma_{C 2}\left(x, y ; x^{\prime} y^{\prime}\right)=\min _{n}\left(\left(x-x^{\prime}+2 n L\right)^{2}+\left(y-y^{\prime}\right)^{2}\right)
\end{gathered}
$$

$n$ is integer

Thus, world function may describe topology of geometry, if one uses a finite world function, but not infinitesimal one.

The world function is an invariant, which does not depend on a choice of coordinate system. It seems to be reasonable to construct a generalized geometry by means of a replacement of the finite world function. It is possible, if all geometrical quantities can be expressed via the world function $G_{E}$.

Usually the Riemannin geometry is constructed by introduction of the metric tensor without a reference to the fact, that the Riemannian geometry is obtained as a result of the $G_{E}$ deformation. As a result of such an approach one cannot consider transformation of dimension $D$ of $G_{E}$. In reality the dimension $D$ of $G_{E}$ depends on the world function $\sigma_{E}$ of $G_{E}$. There exist such deformations of $G_{E}$ (replacements of $\sigma_{E}$ ), that a finite dimension of the generalized geometry becomes to be impossible. For instance, deformation of $G_{E}$ to a discrete geometry $G_{d}$ leads to impossibility of determination of a finite dimension of $G_{d}$. Absence, of a finite dimension in the discrete geometry $G_{d}$ leads to impossibility of a use of the conventional formalism of differential 
geometry. It looks rather natural, because, the differential geometry is based on the supposition of the geometry continuity.

However, the real space-time geometry is discrete in microcosm, and one needs to work with discrete space-time geometry. Let us consider, how geometrical quantities and geometrical objects are defined in term of $\sigma_{E}$ in the proper Euclidean geometry $G_{E}$.

The proper Euclidean geometry is given on the set of points $\boldsymbol{\Omega}$. The geometrical vector ( $\mathrm{g}$ vector) $\mathbf{A B}$ is the ordered set of two points $A$ and $B$. The scalar product (AB.CD) of two g-vectors $\mathbf{A B}$ and $\mathbf{C D}$ is defined by the relation

$$
\begin{gathered}
(\mathrm{AB} . \mathrm{CD})=\sigma_{E}(A, D)+\sigma_{E}(B, C)-\sigma_{E}(A, C)-\sigma_{E}(B, D) \\
|\mathbf{A B}|^{2}=(\mathbf{A B} . \mathbf{A B})=2 \sigma(A, B)
\end{gathered}
$$

$n$ g-vectors $P_{0} P_{1}, P_{0} P_{2}, \ldots P_{0} P_{n}$ are linear dependent, if and only if the Gram's determinant

$$
F_{n}\left(P^{n}\right)=0
$$

where $P^{n}=\left\{P_{0}, P_{1}, \ldots P_{n}\right\}$ and

$$
F_{n}\left(P^{n}\right)=\operatorname{det}\left\|\left(P_{0} P_{i} \cdot P_{0} P_{k}\right)\right\|, i, k=1,2, \ldots n
$$

The Gram's determinant is determined via the scalar products of the g-vectors $P_{0} P_{1}, P_{0} P_{2}, \ldots P_{0} P_{n}$, and it can be determined independently of the existence of linear vector space $L$ in the event space $\Omega$.

The dimension of $G_{E}$ is determined as the maximal number of g-vectors. In order that the maximal number $D$ exists, the following condition must be fulfilled.

$$
\exists P^{D}, F_{D}\left(P^{D}\right) \neq 0, P^{D} \subset \Omega, \quad F_{k}\left(\Omega^{k+1}\right)=0, k>D
$$

In force of (6) relations (7) are restrictions on the form of the world function of the geometry $G_{E}$. These constraints are fulfilled for the $D$-dimensional proper Euclidean geometry 
and for the Riemannian geometry, when the pointset $\Omega=d \Omega$ is infinitesimal. It means, that $\sigma(P, Q)$ is infinitesimal quantity at $\forall P, Q \in d \Omega$.

The discrete geometry $G_{d}$ contains a minimal length $\lambda$. The discreteness of the geometry means that

$$
\left|\rho_{d}(P, Q)\right| \notin(0, \lambda), \quad \forall P, Q \in \Omega
$$

Conventionally, inequality (8) is considered as a constraint on $\Omega$. One obtains a geometry on a lattice. Space-time geometry on a lattice is not uniform and isotropic. It is more correct to consider (8) as a constraint on the world function $\sigma_{d}=\frac{1}{2} \rho_{d}^{2}$. The point set $\Omega$ is manifold $\Omega_{M}$, where the geometry of Minkowski is given. The world function $\sigma_{d}$ is defined by the relation

$$
\sigma_{d}=\sigma_{M}+\frac{\lambda^{2}}{2} \operatorname{sgn}\left(\sigma_{M}\right)
$$

where $\sigma_{M}$ is the world function of the geometry of Minkowski. As far as $\sigma_{d}$ is a function of $\sigma_{M}$, the discrete geometry has the same symmetry as the geometry of Minkowski. Maximal number of linear independent g-vectors is the same in all points of $G_{d}$.

Let $\lambda \ll<1$. Then calculation of the Gram's determinant (6) for g-vectors $P_{0} P_{1}, P_{0} P_{2}, \ldots P_{0} P_{n}$ of the length of the order 1, gives the following result. There are four g-vectors for which the result is of the order 1 . If $\mathrm{n}>4$, then $F_{n} \approx O\left(\lambda^{n-4}\right)$. It means, that for distances $l \cong \lambda$, the discrete spacetime geometry $G_{d}$ coincides approximately with the geometry of Minkowski. However, for $l \cong \lambda$ one needs to develop special formalism of the discrete geometry with indefinite dimension.

Unexpected property of new formalism is multivariance of $G_{d}$ for all vectors. It means, that there are many g-vectors $P_{0} P_{1}, P_{0} P_{1}, \ldots$, which are equivalent to g-vector $Q_{0} Q_{1}$, but g-vectors $P_{0} P_{1}, P_{0} P_{1}, \ldots$ are not equivalent between themselves. Formally such a situation arises, because equality of two g-vectors is defined by two coordinate free equations

$$
\left(P_{0} P_{1} \text { eqv } Q_{0} Q_{1}\right):\left(P_{0} P_{1} \cdot Q_{0} Q_{1}\right)=\left|P_{0} P_{1}\right|\left|Q_{0} Q_{1}\right| \wedge\left|P_{0} P_{1}\right|=\left|Q_{0} Q_{1}\right|
$$


In $G_{E}$ equations (10) determine g-vector $P_{0} P_{1}$ single-valuedly, but in $G_{d}$ one obtains many gvectors $P_{0} P_{1}, P_{0} P_{1}, \ldots$ which are equal to g-vector $Q_{0} Q_{1}$.

At the metric approach there is only one fundamental quantity $\rho(\square P, Q)$. $\square \square \square$ All other geometrical quantities becomes to be derivative. If they exist, they are expressed via metric $\rho(\square P, Q) \square$, or via world function $\sigma=\sqrt{2 \rho}$. It is possible such a situation, when the metric dimension $D$, and the linear vector space do not exist in space-time geometry. However, the spacetime geometry do exist with indefinite dimension $D$ and with absent linear vector space $L$.

Such a situation is perceived hardly. Some researchers are ready to consider a fractional dimension, but not an absence of dimension. Besides, they considered linear dependence of vectors as a property of the linear vector space, because the linear dependence is formulated usually in terms of linear operation in the linear vector space. In reality the linear dependence of vectors is a property of the space-time geometry. It may be formulated in terms of the world function $\sigma$.

The space-time geometry of Minkowski is single-variant with respect to timelike vectors. It is multivariant with respect to spacelike vectors. This result is obtained, if equality of two vectors is defined by two relations (10). It means, that spacelike world line of a tachyon wobbles with infinite amplitude. As a result a single tachyon cannot be detected, but the tachyon gas may be detected by its gravitational field. If the equality of two g-vectors is defined as a coincidence of the vector coordinates, the world line of a tachyon must be smooth. In this case tachyons do not exist, because they have not been discovered experimentally. . Tachyon gas as a dark matter [4,5] and the induced antigravitation [6] tell in favour of the metric conception.

\section{References}

1. Michelson A.A. (1881). The Relative Motion of the Earth and the Luminiferous Ether. American Journal of Science, 22, 120-129.

2. Michelson A.A., Morley A.A., Williams E. (1887). On the Relative Motion of the Earth and the Luminiferous Ether. American Journal of Science, 34, 333-345.

3. Rylov Yu.A. (2014). Metrical conception of the space-time geometry. Int. J. Theor, Phys., 54, iss. 1, 334-339.

4. Rylov Yu.A. (2013). Tahionnyj gaz kak kandidat na tjomnuju materiju [Tachyon gas as a candidate for dark matter]. Vestnik RUDN. Serija «Matematika. Informatika. Fizika» [Herald of RUDN. Series "Mathematics. Informatics. Physics"], iss 2, 159-173. 
Proceedings of International Conference PIRT-2015

5. Rylov Yu.A. (2013). Dynamic equations for tachyon gas. Int. J. Theor. Phys. 52, 133(10), 36833695.

6. Rylov Yu.A. (2012). Induced antigravitation in the extended general relativity. Gravitation and Cosmology, Vol. 18, No. 2, 107-112. 